\title{
Two-Dimensional Moving Blade Row Interactions in a Stratospheric Airship Contra-Rotating Open Propeller Configuration
}

\author{
Zhihao Tang*, Peiqing Liu**, Hao Guo***, Jie Yan**** and Guangchao Li***** \\ School of Aeronautic Science and Engineering, Beihang University, Beijing 100191, China
}

\begin{abstract}
The numerical simulation of two-dimensional moving blade row interactions is conducted by CFD means to investigate the interactions between the front and rear propeller in a stratospheric airship contra-rotating open propeller configuration caused by different rotational speeds. The rotational speed is a main factor to affect the propeller Reynolds number which impact the aerodynamic performance of blade rows significantly. This effect works until the Reynolds number reaches a high enough value beyond which the coefficients become independent. Additionally, the interference on the blade row has been revealed by the investigation. The front blade row moves in the induced-velocity field generated by the rear blade row and the aerodynamic coefficients are influenced when the rear blade row has fast RPMs. The rear blade row moving behind the front one is affected directly by the wake and eddies generated by the front blade row. The aerodynamic coefficients reduce when the front blade row has slow RPMs while increase when the front blade row moves faster than itself. But overall, the interference on the front blade row due to the rear blade row is slight and the interference on the rear blade row due to the front blade row is much more significant.
\end{abstract}

Key words: moving blade row, blade row interaction, contra-rotating open propeller, stratospheric airship

\section{Introduction}

Presently, the possibility of applying a stratospheric airship for high-altitude or long-endurance missions has generated substantial interest in both the commercial and military sectors [1-4]. Usually, the propeller propulsion system is widely applied on the stratospheric airships because it is the most efficient means that cannot be matched by a turbojet or turbofan [5]. However, the special flight conditions of stratospheric airship propellers, especially the much smaller advance ratio and lower Reynolds number than conventional propellers, bring many troubles on the propulsion efficiency. In this case, the performance of a generic isolated stratospheric airship contra-rotating open propeller (CROP) system was investigated by Liu [6-8] in order to find a new way to solve the efficiency problem. As expected, Liu's measurements revealed trends of reduction of thrust and efficiency of the front propeller but increase of thrust and efficiency of the rear propeller compared to the single-rotating open propeller (SROP), and overall increase of thrust and efficiency. It indicated the advantages on efficiency of CROP system at small advance ratios and low Reynolds numbers.

However, for the early conceptual stages of CROP design, "engineering codes" are still the preferred method for analysis because this approach can trim many months off of the design process compared with computational fluid dynamics (CFD) calculations or wind tunnel tests [9]. In order to develop the codes, the interaction between the front and rear propellers has to be revealed due to its important influence on the overall performance of CROPs. Therefore, the investigation of combination parameters, such as rotational speed ratio or spacing distance of two propeller, is necessary to the CROP design and application. Some direct or indirect related studies can be found. For example, in 1940s, Gray's experimental study [10] found that the peak efficiency of a CROP was not seriously affected by small changes in blade
This is an Open Access article distributed under the terms of the Creative Commons Attribution Non-Commercial License (http://creativecommons.org/licenses/by$\mathrm{nc} / 3.0 /$ which permits unrestricted non-commercial use, distribution, and reproduction in any medium, provided the original work is properly cited. 
angle or changes in rotational speed of the rear propeller. Bartlett [11] conducted the experiment of effect on the propulsive efficiency of locking or windmilling one propeller for both tractor and pusher positions. It indicated that a CROP with one propeller disabled resulted in a reduction of total propeller efficiency. In 1987 and 1994, Harrison [12] and Shin [13] conducted a measurement upstream, in between, and downstream of the CROP system using laser Doppler velocimeter (LDV) and three-dimensional hotwire anemometry respectively, which proved the recovery by the downstream propeller of the swirl velocity imparted to the flow by the upstream propeller was very evident. In addition, the particle image velocimetry (PIV) apparatus was also applied to investigate the complex interaction flowfield of a generic contra-rotating open rotor model at wind-tunnel scale by Stürmer [14]. Nevertheless, these experimental studies were concentrated on conventional aircraft at relatively low-altitude and far away from enough to develop an accurate and efficient codes. More systematic and parametric investigation related to high-altitude aircraft was imperative.

As the CFD calculation methods have been improved significantly in last decades, numerical simulation become an efficient means in the CROP rotor-rotor interaction investigation. However, due to the relative motion of the front and rear rotor the flow in a CROP is of high unsteady nature. The calculation of three-dimensional CROP configuration by CFD means would cost huge amount of time and computing resources. As the previous similar investigation [15], the infinite two-dimensional blade-row model of propeller blade elements often used to be regarded as a simple and efficient means to reveal blade-to-blade interactions rather than complex three-dimensional numerical simulation. Former two-dimensional cascade investigation was primarily aimed at the interactions between rotor and stator blade rows in turbomachines. Some of them could be taken as references into two-dimensional CROP bladeto-blade interaction investigation. For example, Fontanals [16] studied the rotor-stator interaction phenomenon in a moving cascade of airfoils in turbomachinery under design and off-design conditions. Arko [17] in 2013 used a Reynolds-averaged Navier-Stokes finite volume flow solver to simulate the flowfields around a two-dimensional linear turbine cascade model at a Reynolds number of 25,000. An unsteady multistage CFD prediction was also conducted by Lipfert [18] in recent years to allow detailed insight and understanding of the blade row interactions in a low pressure turbine. Walther [19] developed the discrete adjoint equations for a turbomachinery Reynolds-averaged Navier-Stokes solver and proposed a framework for fully automatic gradient-based constrained aerodynamic shape optimization in a multistage turbomachinery environment.

However, the moving blade row model was seldom applied in the rotor-rotor interaction of a CROP configuration at low Reynolds number and small advance ratio. In this paper, the two-dimensional moving blade row interactions have been simulated by CFD means to investigate the interactions between the front and rear propeller in a stratospheric airship CROP configuration caused by different rotational speeds.

\section{Numerical Method and Validation}

\subsection{Geometry and Grid Generation}

The prototype of the CROP used in numerical simulation is reported in detail in reference [7]. Therefore, the twodimensional CROP model can be represented with two opposite moving blade rows, as shown in Fig. 1. The blade element spacing $s$ represents the distance between two adjacent blades of a propeller along a certain circular arc (see Fig. 2). So it is related to the arc perimeter, radial location, and the propeller blade number, as defined in Eq. (1).

$$
s=\frac{x \pi D}{N_{B}}
$$

where $x$ is the proportional radius of blade element, $D$ is the diameter of each propeller disk, and $N_{B}$ is the blade number of each propeller. The distance $d$ between two blade rows represents the spacing between the front and rear propeller. The pitch angle $\theta$ defined in Fig. 1 is the summation of the installation angle and the local twist angle.

$$
\theta=\varphi+\chi
$$

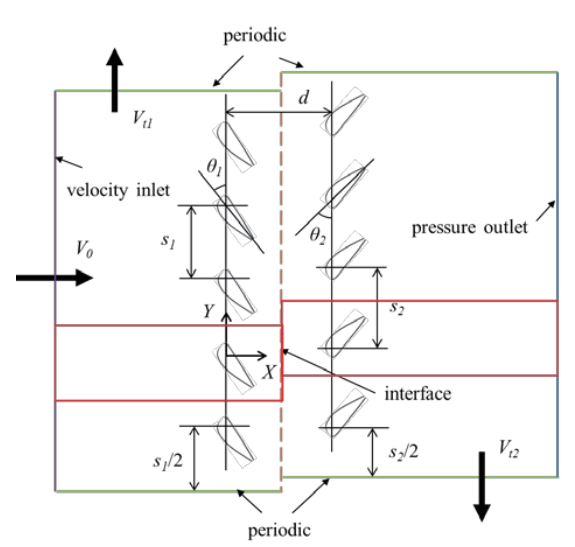

Fig. 1. General drawing of the setup and boundary condition imposed in the numerical modeling 
where $\varphi$ is the installation angle of blade and $\chi$ is the local twist angle of the blade element. The airfoils of the blade elements is S1223 of which the chord length, max thickness, and pitch angle depend on their radial location on the propeller blades. Due to the longest chord length, the blade element at radial location of $x=0.50$ has been chosen for simulation in this paper. The parameters of the CROP model and the blade element at $x=0.50$ are shown in Table 1 . Other details of the related wind tunnel tests have also been reported in reference [7].

Structured meshing technique is adopted establishing sliding mesh configuration as the analysis is unsteady as per CFD code [20]. In order to facilitate meshing the grid, just one front and one rear blade elements are applied (red box in Fig. 1) and the period condition is used. Other setting of boundary conditions can be seen in Fig. 1. The mesh near the airfoil surfaces has been refined due to the complex flowfields at low Reynolds number. Furthermore, a static middle area is added between the front and rear rotor fluids to ensure the flow information well transmitted from the front rotor fluid to the rear. Figure 3 shows the details of the

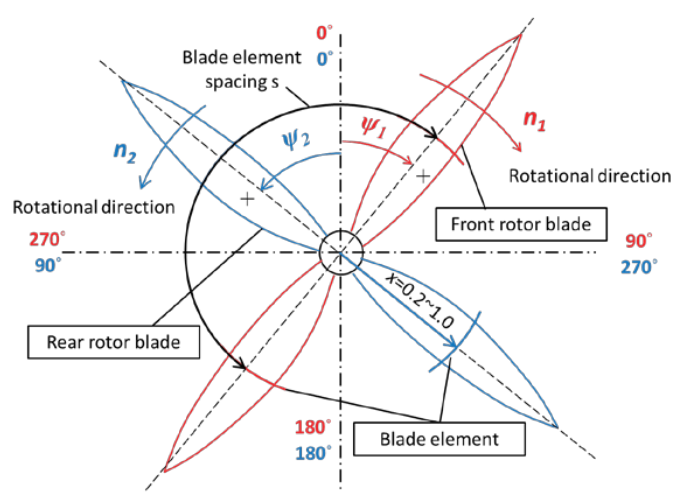

Fig. 2. Definition of the blade row parameters

Table 1. The parameters of the CROP model and the blade element at $x=0.50$

\begin{tabular}{|c|c|c|}
\hline \multicolumn{3}{|c|}{ CROP model } \\
\hline Propeller & Front & Rear \\
\hline Distance, $\mathrm{m}$ & \multicolumn{2}{|c|}{0.15} \\
\hline Diameter, $\mathrm{m}$ & 0.75 & 0.75 \\
\hline Installation angle, deg & 28.3 & 28.3 \\
\hline Blade number & 2 & 2 \\
\hline \multicolumn{3}{|c|}{ Blade element at $x=0.50$} \\
\hline Blade element & Front & Rear \\
\hline Airfoil & S1223 & S1223 \\
\hline Chord length, m & 0.05353 & 0.05353 \\
\hline Max thickness, m & 0.006133 & 0.006133 \\
\hline Twist angle, deg & 9.43 & 9.43 \\
\hline Pitch angle, deg & 37.73 & 37.73 \\
\hline
\end{tabular}

mesh near the moving blade rows.

\subsection{Unsteady Calculation Setup}

Two-dimensional unsteady Reynolds-averaged NavierStokes equation is solved by means of a commercial CFD code, ANSYS FLUENT 14.0 [20]. To simulate the moving blade row interactions in the CROP configuration, a constant velocity is applied at the inlet. The transverse velocity of the blade row ( $V_{t}$ in Fig. 1) represents the tangential speed of the blade element. So it depends on the rotational speed and the element radial location, as defined in Eq. (3).

$$
V_{t}=\frac{x \pi n D}{60}=x \pi n_{s} D
$$

where both $n$ and $n_{s}$ are the rotational speed, but the units of them are RPM and round per second, respectively. A non-slip condition is specified for the flow at the moving wall boundaries of the rotor blade. A periodic condition is applied to the rotor fluid and a static pressure condition is imposed at the outlet of the rear rotor fluid.

The turbulence is modeled using the SST $k$ - $\omega$ model with low Reynolds number corrections, since it is a good option due to its accurate performance both in boundary layer and in wake flow modeling [21]. Experimental turbulence intensity of $0.08 \%$ is applied at the inlet velocity boundary condition. As previous numerical investigation of similar problems [16], the unsteady formulation used is a second-order implicit velocity formulation, and a pressure-based solver is chosen (the effect of Mach number could be neglected here [6-8]). The SIMPLE pressure-velocity coupling algorithm is used and second order scheme discretization is selected for the numerical simulation. The interface between the front rotor and the middle area, as well as between the middle area and the rear rotor, is set to a sliding mesh, in which the relative position between two areas is updated every time step. The

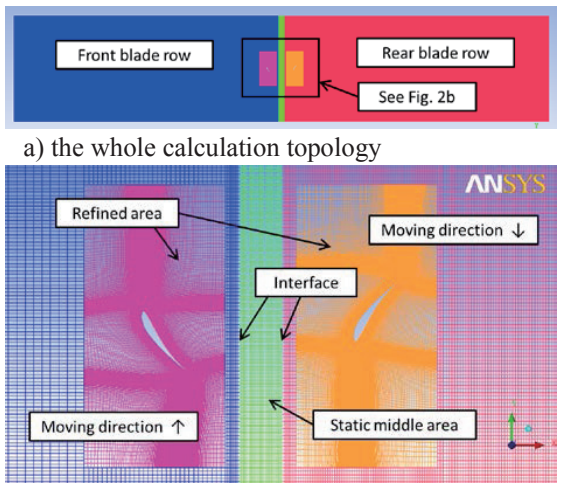

b) the refined mesh near the airfoil surfaces

Fig. 3. Mesh of the whole calculation topology and the refined mesh near airfoils 
maximum number of iterations for each time step is set to 40 in order to reduce all computed residuals under $1 \times 10^{-5}$. Due to the unsteady nature of the flow, it is required that the whole flow domain is affected by the unsteady fluctuations. In order to check the aforementioned setting, a force monitor is recorded at the rotor blade element surfaces.

\subsection{Turbulence Model Validation, Mesh sensitivity, and Time Step Selection}

Before the blade row interaction numerical simulation, the SST $k$ - $\omega$ model needs validation via the S1223 airfoil calculation at various low Reynolds numbers. The C-block is applied with a $y^{+}=1 / 2$ in the validation. The calculated lift coefficients are compared with the experimental data [22,23], as shown in Fig. 4. It can be found that the CFD predictions for the linear part (before stall angle of attack (AOA)) of the lift curve are very accurate regardless of the Reynolds number. At $R e=80,000$, the airfoil lift coefficients beyond the stall AOA are over-predicted, while that are less-predicted at $R e=140,000$ or $R e=200,000$. The difference between the CFD predictions and the experimental data in the poststall regime is also found in many other similar researches. In general, it is fair to say that the prediction of separated flow in the poststall region still remains a challenging frontier in CFD [24]. In addition, the pitch angle of the blade element applied in this paper is fixed at $37.73^{\circ}$. So the actual AOA of blade element is around $5^{\circ}$ at $J=1.0$. It means the simulation results would be reliable as the SST $k$ - $\omega$ model is applied at low Reynolds number in this paper.

For evaluating the mesh sensitivity and deciding the appropriate time step three 2-D grids (coarse, medium, and fine) and three sets of time step ( $\lambda=320, \lambda=400$, and $\lambda=500$, $\lambda$ is the calculation times per circle of propeller) have been used (Table 2). The boundary layer around the rotor blades is modeled with a $y^{+}=1 / 2$. Some aerodynamic parameters of

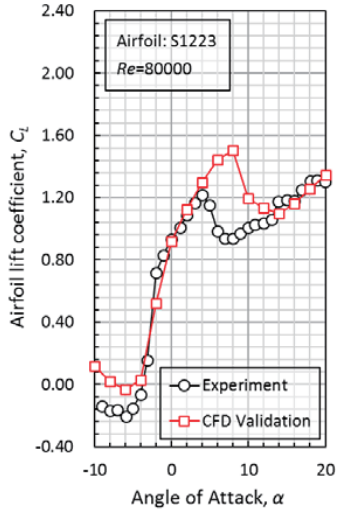

a) $\operatorname{Re}=80,000$

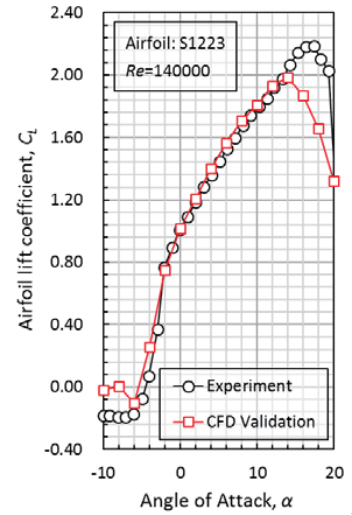

b) $\operatorname{Re}=140,000$

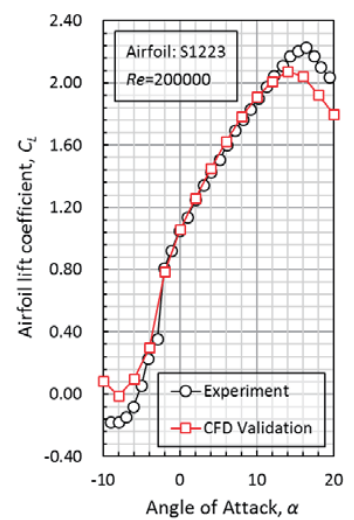

c) $\operatorname{Re}=200,000$

Fig. 4. Lift curves of the S1223 airfoil at various Reynolds number

Table 2. Mesh and time step sensitivity test

\begin{tabular}{ccccccc}
\hline \multicolumn{7}{c}{ Time step $\lambda=320$} \\
\hline Mesh & Cell number & $y^{+}$ & $d C_{T 1}$ & $d C_{T 2}$ & $d C_{Q 1}$ & $d C_{Q 2}$ \\
\hline Coarse & 218,700 & $1 / 2$ & 0.16058 & 0.20013 & 0.03074 & 0.03600 \\
Medium & 341,540 & $1 / 2$ & 0.16064 & 0.20111 & 0.03072 & 0.03610 \\
Fine & 534,924 & $1 / 2$ & 0.16068 & 0.20182 & 0.03063 & 0.03571 \\
\hline \multicolumn{7}{c}{ Time step $\lambda=400$} \\
\hline Mesh & Cell number & $y^{+}$ & $d C_{T 1}$ & $d C_{T 2}$ & $d C_{Q 1}$ & $d C_{Q 2}$ \\
\hline Coarse & 218,700 & $1 / 2$ & 0.16061 & 0.20014 & 0.03074 & 0.03601 \\
Medium & 341,540 & $1 / 2$ & 0.16946 & 0.20406 & 0.03266 & 0.03648 \\
Fine & 534,924 & $1 / 2$ & 0.16976 & 0.20454 & 0.03285 & 0.03667 \\
\hline \multicolumn{7}{c}{ Time step $\lambda=500$} \\
\hline Mesh & Cell number & $y^{+}$ & $d C_{T 1}$ & $d C_{T 2}$ & $d C_{Q 1}$ & $d C_{Q 2}$ \\
\hline Coarse & 218,700 & $1 / 2$ & 0.16066 & 0.20015 & 0.03075 & 0.03601 \\
Medium & 341,540 & $1 / 2$ & 0.17086 & 0.20479 & 0.03372 & 0.03670 \\
Fine & 534,924 & $1 / 2$ & 0.17127 & 0.20497 & 0.03391 & 0.03694 \\
\hline
\end{tabular}


rotor blade element should be introduced here.

Advance ratio of the propeller:

$J=\frac{V_{0}}{n_{s} D}$

Thrust coefficient of blade element:

$d C_{T}=-\frac{F_{x}}{\rho n_{s}^{2} D^{4}}$

Torque coefficient of blade element:

$$
\begin{aligned}
& d C_{Q 1}=-\frac{F_{y 1}}{\rho n_{s 1}^{2} D_{1}^{5}} \cdot \frac{x D_{1}}{2}=-\frac{x F_{y 1}}{2 \rho n_{s 1}^{2} D_{1}^{4}} \\
& d C_{Q 2}=\frac{x F_{y 2}}{2 \rho n_{s 2}^{2} D_{2}^{4}}
\end{aligned}
$$

The nominal efficiency of blade element:

$$
d \eta=J \cdot \frac{d C_{T}}{2 \pi \cdot d C_{Q}}
$$

where $V_{0}$ is the advance speed of the propeller or the wind speed in the wind tunnel tests, $\rho$ is the air density, and $F_{x}$ and $F_{y}$ are the force calculated along the $X$-direction and the $Y$-direction, respectively. The subscripts 1 and 2 represent the front and rear blade elements, respectively. During the validation process, the propeller RPMs are 1500 and the
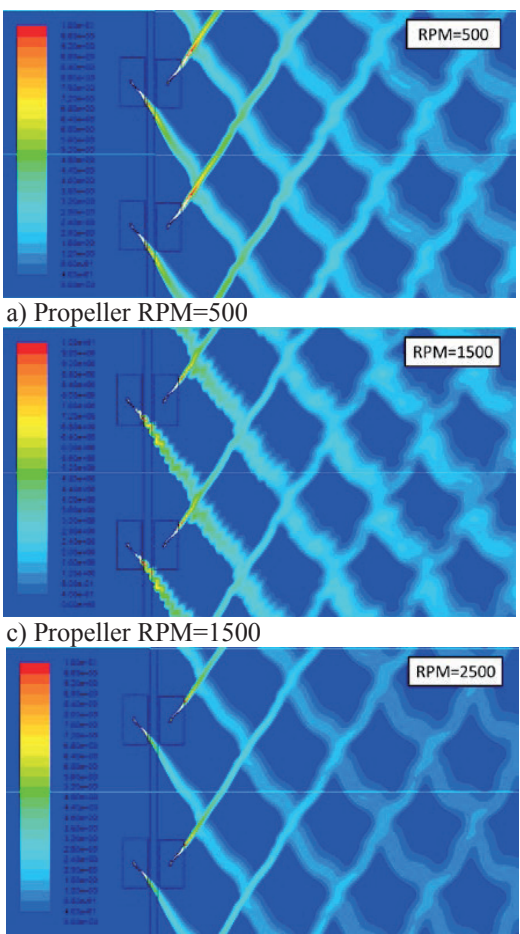

e) Propeller RPM=2500 advance ratio is 1.0 .

The calculated results summarized in Table 2 demonstrates that: 1) when the coarse mesh is applied, the aerodynamic parameters are less-predicted than medium and fine mesh although they are little influenced by the different time steps. 2) when the medium and fine mesh are applied, the values of aerodynamic parameters trend to be close from the time step of $\lambda=320$ to $\lambda=500$. 3) there is few difference between the medium and fine mesh applied as the time step of $\lambda=400$ and $\lambda=500$. As aforementioned, it can be concluded that the medium mesh with $\lambda=400$ can be employed in the simulations presented in this paper.

\section{Results and Discussions}

\subsection{Effect of Reynolds Number}

The blade row interactions with different propeller RPMs (the same RPM held by the front and rear blade elements) are investigated in this part. The rotational speed is a key factor to affect the Reynolds number of CROP system. In the two-dimensional blade row configuration, the Reynolds number of a blade element can be defined as

$$
\operatorname{Re}_{x}=\frac{\rho b \sqrt{V_{0}^{2}+V_{t}^{2}}}{\mu}
$$
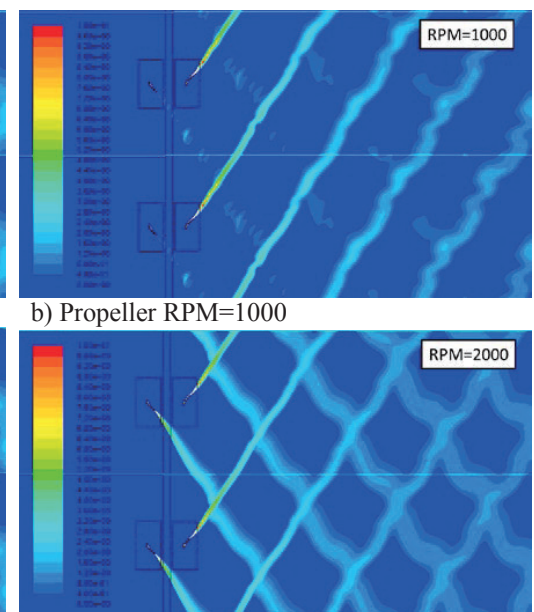

d) Propeller RPM=2000

Fig. 5. Computed turbulence intensity contour fields at several rotational speeds 
where $b$ is the chord length of blade element and $\mu$ is the air dynamic viscosity coefficient. As the previous tests [6-8], the peak efficiency occurred when the advance ratio is about 1.0. This value is applied in this simulation. The conditions of numerical simulations are introduced in Table 3.

A numerical study of a flow behavior in the wake generated by the front moving rotor blades and its corresponding interaction with the rear moving rotor blades is conducted here by means of modeling the flow turbulence intensity on the wake (see Fig. 5). The figure shows the behavior pattern of the wake is different for each rotor frequency analyzed. It can be observed that, at operating condition of RPM=500, the boundary layer seems to be thick and the laminar flow easily goes transition on the upper surfaces of front blade elements due to the low Reynolds number. As contrast, the wake presents a vortex shedding with small eddies at RPM=1000. The characteristic length of eddies trends to be larger at $\mathrm{RPM}=1500$ and then the vortex shedding starts to disappear at $R P M=2000$ and $R P M=2500$. It indicates the turbulence intensity pattern is strongly dependent on the Reynolds number of rotor blade elements. For the rear rotor blade, the boundary layer is also thick but no vortex shedding on the blade surface during all the computed RPM range under the interference from the front rotor. It means the difference of wake behavior pattern due to Reynolds number is found exist mainly in the wake of front rotor blades.

The wake behavior pattern has been changed due to the Reynolds number so that the aerodynamic force on the blade elements of course is different at varying RPMs. Figure 6 shows the computed thrust coefficient, torque coefficients, and nominal efficiency of blade elements at the phase position $\left(\psi_{1}+\psi_{2}\right)$ from $0^{\circ}$ to $360^{\circ}$. The definition of the phase position is introduced in Fig. 2. Due to the same rotational speed, the phase position of front and rear blade elements should be always equal at the same time. During all the simulation process, the initial phase position of front and rear blade elements are both $0^{\circ}$. According to Fig. 6, the aerodynamic coefficients can be found fluctuant at $\mathrm{RPM}=1000$ and $\mathrm{RPM}=1500$ owing to the vortex shedding on the blade surface. Additionally, the figure indicates the coefficients of two-dimensional stratospheric airship CROP blade rows are highly dependent on Reynolds number.
These coefficients increase with the increasing of Reynolds number. The impact of Reynolds number is significant until it reaches a high enough value beyond which the coefficients become Reynolds number independent. It indicates, in terms of the performance of CROP on a stratospheric airship, the Reynolds number which the airship is flying at is an important factor to be considered.

\subsection{Interference on Front Rotor Blade Elements}

Different from the SROP rotating in a free flowfield, the front rotor rotates in the induced-velocity field generated by the rear rotor in a CROP configuration. When the two rotors of CROP stay close with each other, the interference obviously exists to affect the aerodynamic performance of each rotor. Some cases are calculated in which the front blade element (at $x=0.50$ ) RPM remains 1500 while the rear blade element RPM changes from 0 to 2500 to investigate the interference on the two-dimensional front blade rows. Table 4 shows the time-averaged aerodynamic coefficients of the front blade element at the phase position $\left(\psi_{1}+\psi_{2}\right)$ from $0^{\circ}$ to $360^{\circ}$. The performance of SROP blade element is also introduced in the table as a comparison. Compared with SROP, the performance of the front blade element has no obvious change when the rear blade element RPM is 0 and 500. It indicates the induced-velocity field generated by rear blade elements at slow rotational speed is not strong enough to impact the performance of front blade elements. As the rear blade element RPM increases, the thrust and torque coefficients of the front blade element increase except when the rear blade element RPM is 2000. It means the forces on the front blade element are influenced more significantly with the induced-velocity flied getting stronger. Under this circumstance, the nominal efficiency of front blade elements which under the interference by rear blade rows is slightly low or almost equal to the SROP.

Figure 7 shows the pressure coefficients on the upper and lower surface of the front blade elements in function of the phase position $\left(\psi_{1}+\psi_{2}\right)$. It can be found that the value of pressure coefficient of the front blade element depends on the strength of the induced-velocity field the rear blade row creates. The variation of pressure coefficients have different

Table 3. The numerical simulation conditions

\begin{tabular}{ccccc}
\hline Propeller RPM & $V_{t}, \mathrm{~m} / \mathrm{s}$ & $J$ & $V_{0}, \mathrm{~m} / \mathrm{s}$ & $R e_{0.5}$ \\
\hline 500 & 9.82 & 1.0 & 6.25 & $0.43 \times 10^{5}$ \\
1000 & 19.63 & 1.0 & 12.50 & $0.85 \times 10^{5}$ \\
1500 & 29.45 & 1.0 & 18.75 & $1.28 \times 10^{5}$ \\
2000 & 39.27 & 1.0 & 25.00 & $1.71 \times 10^{5}$ \\
2500 & 49.09 & 1.0 & 31.25 & $2.13 \times 10^{5}$ \\
\hline
\end{tabular}


regulation due to different rear blade element RPMs. For example, the pressure coefficients under the interference of the rear blade elements at $R P M=1000, R P M=1500$, or $\mathrm{RPM}=2500$ fluctuate with the variation of the phase position. Additionally, according to the figure, the rear blade element pressure field more severely affects the lower surface instead of the upper surface. On the other hand, compared with SROP, the pressure coefficient of the front blade element, no matter on the upper or lower surface, is a bit larger at rear $\mathrm{RPM}=0$ or $\mathrm{RPM}=500$ while the coefficient is smaller at other rear blade element RPMs. Due to the interference from the rear blade elements, the pressure coefficient of the upper surface reduces with the increasing of the rear blade element RPM. The similar phenomenon can be observed on the lower surface. However, although the upper and lower surface are both influenced by the rear blade elements, the overall forces on the front blade element have not changed significantly (see Table 4).

\subsection{Interference on Rear Rotor Blade Elements}

Similarly, the interference on the rear blade rows has been investigated via remaining the value of rear blade element RPM at 1500 unchanged. Table 5 shows the time-averaged aerodynamic coefficients of the rear blade element at the phase position $\left(\psi_{1}+\psi_{2}\right)$ from $0^{\circ}$ to $360^{\circ}$. Different from the front blade elements, the rear blade elements installed behind the front ones so that to be influenced directly by the wake and eddies of the front blade elements. Therefore, the obvious difference can be observed in the table under varying calculation conditions. Compared with SROP, the performance of the rear blade element is weakened when the front blade element RPM is slow (at 0 or 500) while the
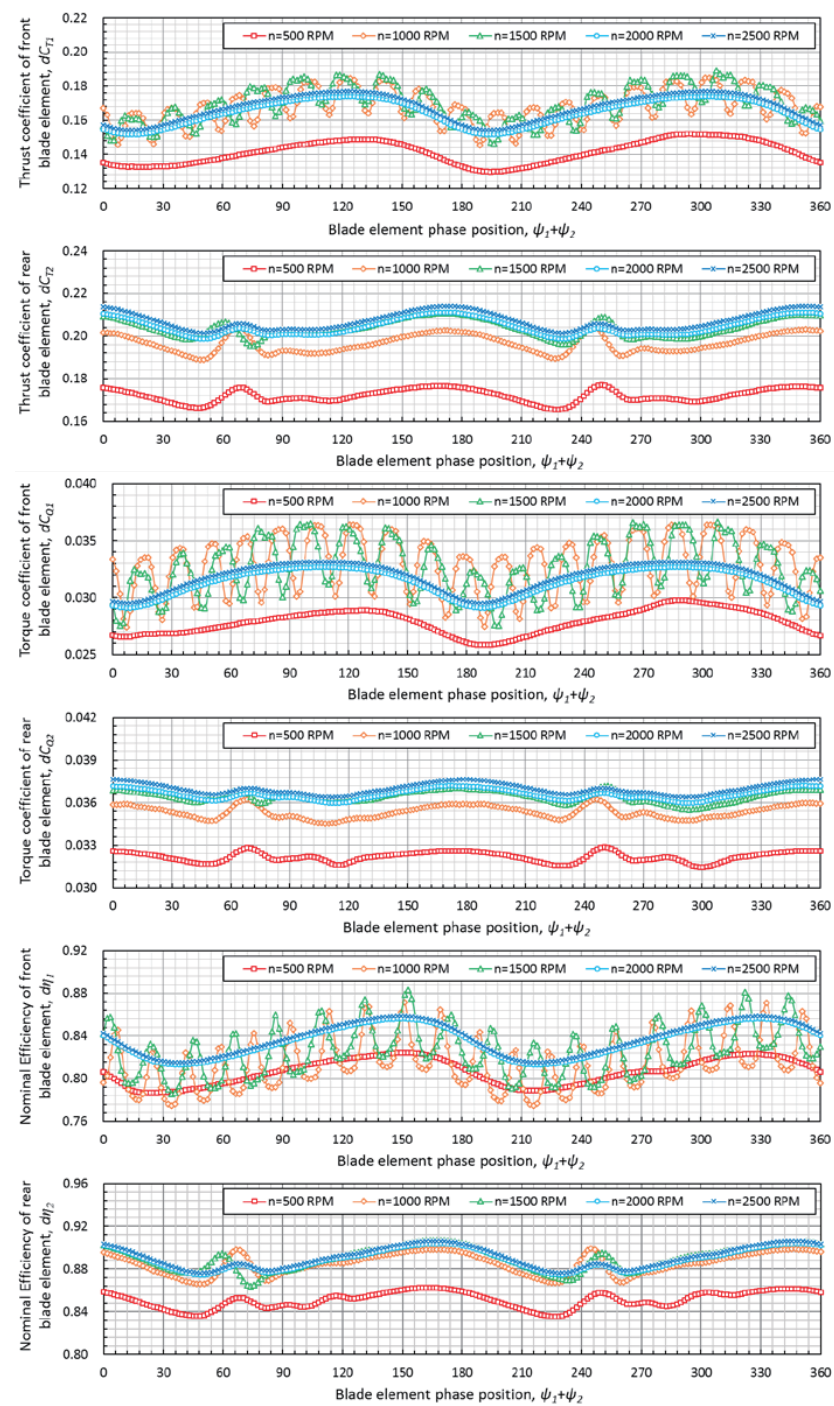

Fig. 6. Computed thrust coefficient, torque coefficients, and nominal efficiency of blade elements 
force on the rear blade element at fast RPMs (over 1500) is improved. It indicates whether the wake or eddies of the front blade element can improve the performance of the rear blade element depends on the strength and pattern of the wake the front blade element creates. As the front blade element RPM increases, the thrust, torque coefficients, and the nominal efficiency of the rear blade element significantly increase. It means the wake strength and pattern at fast front blade element RPMs offers benefits to the rear blade element performance improvement.

Figure 8 shows the pressure coefficients on the upper and lower surface of the rear blade elements in function of the phase position $\left(\psi_{1}+\psi_{2}\right)$. It can be found that the force on the rear blade element is closely related to the phase position. Different from Fig. 7, the coefficients in Fig. 8 have no fluctuant at any phase position. However, there are some other special points should be paid attentions: the sharply variation of the pressure coefficient on the upper or lower surface of the rear blade element when the $\left(\psi_{1}+\psi_{2}\right)$ is about $180^{\circ}$. That's probably the moment that the eddies created by the front blade element just drop on the upper surface of the rear blade element. Moreover, the wake or eddies from the front blade elements seem to influences the upper surfaces of the rear blade elements more severely than the lower surfaces. Compared with SROP, the pressure coefficient on the upper surface of the rear blade element at small front

Table 4. Time-averaged aerodynamic coefficients of front blade elements

\begin{tabular}{ccccc}
\hline Front rotor RPM & Rear rotor RPM & $d C_{T I}$ & $d C_{P I}$ & $d \eta_{1}$ \\
\hline & 0 & 0.16003 & 0.03064 & 0.83111 \\
& 500 & 0.16065 & 0.03073 & 0.83193 \\
1500 & 1000 & 0.16883 & 0.03259 & 0.82555 \\
& 1500 & 0.16937 & 0.03264 & 0.82669 \\
& 2000 & 0.16071 & 0.03072 & 0.83233 \\
& 2500 & 0.17432 & 0.03378 & 0.82232 \\
\hline \multicolumn{2}{c}{ SROP $(\mathrm{RPM}=1500)$} & 0.16066 & 0.03074 & 0.83180 \\
\hline
\end{tabular}

Table 5. Time-averaged aerodynamic coefficients of rear blade elements

\begin{tabular}{ccccc}
\hline Front rotor RPM & Rear rotor RPM & $d C_{T 2}$ & $d C_{P 2}$ & $D \eta_{2}$ \\
\hline 0 & & 0.13838 & 0.02685 & 0.81413 \\
500 & & 0.14994 & 0.02922 & 0.81581 \\
1000 & \multirow{2}{*}{1500} & 0.16017 & 0.03074 & 0.82890 \\
1500 & & 0.20425 & 0.03651 & 0.89027 \\
2000 & & 0.23067 & 0.03969 & 0.92488 \\
2500 & & 0.23928 & 0.04077 & 0.93294 \\
\hline \multicolumn{2}{l}{ SROP $(\mathrm{RPM}=1500)$} & 0.16066 & 0.03074 & 0.83180 \\
\hline
\end{tabular}

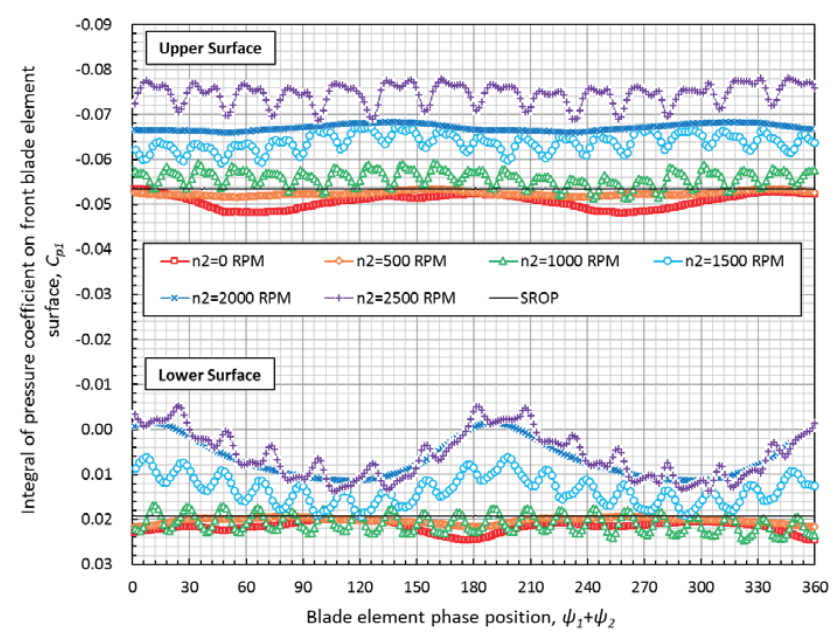

Fig. 7. Integral of pressure coefficient of front blade element surface 


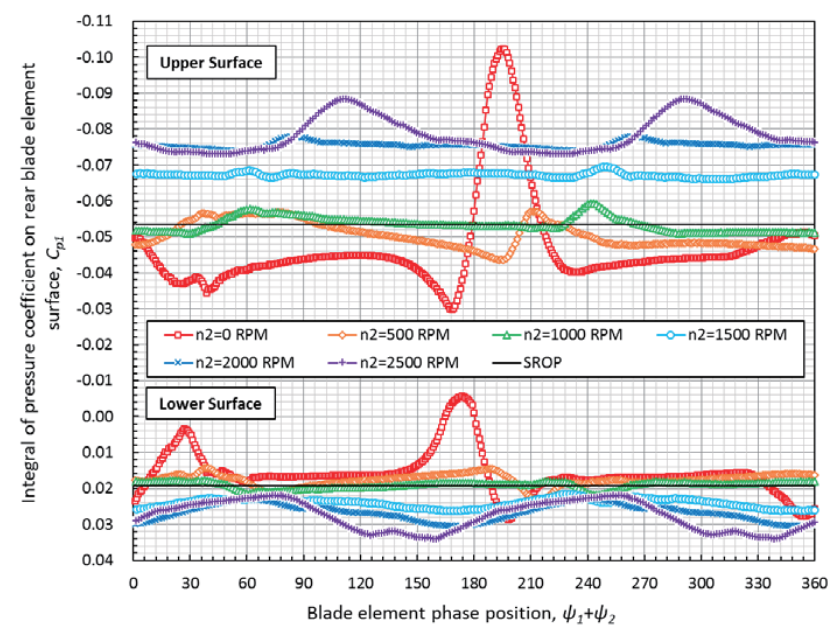

Fig. 8. Integral of pressure coefficient of rear blade element surface

RPMs is smaller. Due to the interference from the wake of front blade elements, the pressure coefficient on the upper surface reduces with the increasing of the front blade element RPM. However, the regulation on the lower surface of the rear blade element is totally on the contrary. The pressure coefficient on the lower surface of the rear blade element at small front RPMs is bigger than that of SROP blade element. It increases with the increasing of the front blade element RPM. The opposite impact on the upper and lower surface is the reason why the performance of the rear blade varies greatly with the change of the front blade RPM.

\section{Conclusions}

The numerical investigation of two-dimensional moving blade rows (at $x=0.50$ ) is conducted in this paper to illustrate the interactions between two rotors caused by varying rotational speed in a stratospheric airship CROP configuration. Maybe the pattern of blade rows flowfield cannot totally represent the whole three-dimensional CROP flowfield, but this investigation will significantly simplify the difficulty of research process by CFD means and also reveal some useful regulations.

1) The wake and pattern of the front blade row would be affected differently at varying Reynolds number while the wake of the rear blade row would be affected slightly. Accordingly, the aerodynamic coefficient of the blade element would increase with the increasing of the Reynolds number. The effect of Reynolds number is significant until it reaches a high enough value beyond which the coefficients become independent.
2) In terms of the front blade row at a fixed RPM (1500 in this paper), it moves and is affected in the induced-velocity field generated by the moving rear blade row. The lower surface is influenced more severely than the upper surface. The aerodynamic coefficients are almost unchanged when the RPM of the rear blade row is slow, and then increase with the increasing of the rear blade row RPM. But overall, the interference on the front blade row due to the rear blade row is relatively slight.

3) In terms of the rear blade element at a fixed RPM (1500 in this paper), it moves behind the front blade row and is affected directly by the wake and eddies generated by the front blade row. The aerodynamic coefficients reduce when the RPM of front blade row is slow, and then increase when the front blade row moves faster than itself. The upper surface is influenced more severely than the lower surface. Additionally, the opposite regulation would be observed respectively on the upper and lower surfaces.

Besides the rotational speed, some other investigation of combination parameters in a stratospheric airship CROP configuration is on the way. All the aforementioned research proves the interference between two rotors and provide a reference for developing the "engineering note" to simply predict the performance of CROP system at small advance ratio and low Reynolds number.

\section{Acknowledgement}

This work was supported by the National Natural Science Foundation of China (Grant No. 11272034), the China-EU Aeronautical Science \& Technology Cooperation Project DRAGY. 


\section{References}

[1] Colozza, A., "Initial Feasibility Assessment of a High Altitude Long Endurance Airship", NASA/CR-2003-212724, 2003.

[2] Moomey, E. R., “Technical Feasibility of Loitering Lighter-than-Air Near-Space Maneuvering Vehicles”, M.S. Dissertation, U.S. Air Force Inst. Of Technology Rept. ADA437762, Wright-Patterson AFB, OH, March 2005.

[3] Li, Y., Nahon, M. and Sharf, I., "Airship dynamics modeling: A literature review", Progress in Aerospace Sciences, Vol. 47, 2011, pp. 217-239. DOI: 10.1016/j. paerosci.2010.10.001

[4] Shen, J. Q., Pan. C., Wang, J. J., Yi, H. M. and Li, T., “ReynoldsNumber Dependency of Boundary-Layer Transition Location on Stratospheric Airship Model", Journal of Aircraft, Vol. 52, No. 4, 2015, pp. 1355-1359. DOI: 10.2514/1.C032971

[5] Carichner, G. E. and Nicolai, L. M., Fundamentals of Aircraft and Airship Design (Volume 2: Airship Design and Case Studies), AIAA Education Series, AIAA, New York, 2013, pp. 151-195.

[6] Liu, P., Tang, Z., Chen, Y. and Guo, H., "Experimental Feasibility Assessment of Stratospheric Airship CounterRotating Propellers", AIAA 53rd Aerospace Sciences Meeting, Kissimmee, Florida, 2015, AIAA Paper 2015-1029. DOI: 10.2514/6.2015-1029

[7] Tang, Z., Liu, P., Chen, Y. and Guo, H., "Experimental Study of Contra-Rotating Propellers for High-Altitude Airships", Journal of Propulsion and Power, Vol. 31, No. 5, 2015, pp. 1491-1496. DOI: 10.2514/1.B35746

[8] Tang, Z., Liu, P., Sun, J., Chen, Y., Guo, H. and Li, G., "Performance of Contra-Rotating Propellers for Stratospheric Airship", International Journal of Aeronautical and Space Sciences, Vol. 16, No. 4, 2015.

[9] Whitmore, S. A. and Merrill, R. S., "Nonlinear Large Angle Solutions of the Blade Element Momentum Theory Propeller Equations", Journal of Aircraft, Vol. 49, No. 4, 2012, pp. 1126-1134. DOI: 10.2514/1.C031645

[10] Gray, W. H., "Wind Tunnel Test of Dual-Rotating Propellers with Systematic Differences in Number of Blades, Blade Setting and Rotational Speed of Front and Rear Propellers", NACA ARR No. L4E22 (WR L-80), 1944.

[11] Bartlett, W. A., "Wind-Tunnel Tests of a Dual-Rotating Propeller Having One Component Locked or Windmilling", NACA ARR No. L5A13a (WR L-214), 1945.

[12] Harrison, G. L. and Sullivan, J. P., "Measurement of a Counter Rotation Propeller Flowfield Using a Laser Doppler Velocimeter", AIAA 25 th Aerospace Sciences Meeting, Reno,
Nevada, 1987, AIAA Paper 1987-0008. DOI: 10.2514/6.1987-8

[13] Shin, H., Whitfield, C. E. and Wisler, D. C., "RotorRotor Interaction for Counter-Rotating Fans, Part 1: ThreeDimensional Flowfield Measurements", AIAA Journal, Vol. 32, No. 11, 1994, pp. 2224-2233. DOI: 10.2514/3.12281

[14] Stürmer, A., Gutierrez, C. O. M., Roosenboom, E. W. M., Schröder, A., Geisler, R., Pallek, D., Agocs, J. and Neitzke, K., "Experimental and Numerical Investigation of a Contra Rotating Open-Rotor Flowfield", Journal of Aircraft, Vol. 49, No. 6, 2012, pp. 1868-1877. DOI: 10.2514/1.C031698

[15] Okulov, V. L., Sorensen, J. N. and Wood D. H., "The rotor theories by Professor Joukowsky: Vortex theories", Progress in Aerospace Sciences, Vol. 73, 2015, pp. 19-46. DOI: 10.1016/j.paerosci.2014.10.002

[16] Fontanals, A., Coussirat, M., Guardo, A. and Egusquiza, E., "Detailed study of the rotor-stator interaction phenomenon in a moving cascade of airfoils", IOP Conference Series: Earth and Environmental Science, Vol. 12, 2010. DOI: 10.1088/1755-1315/12/1/012089

[17] Arko, B. M. and McQuilling, M., “Computational Study of High-Lift Low-Pressure Turbine Cascade Aerodynamics at Low Reynolds Number", Journal of Propulsion and Power, Vol. 29, No. 2, 2013, pp. 446-459. DOI: 10.2514/1.B34576

[18] Lipfert, M., Habermann, J., Rose, M. G., Staudacher, S. and Guendogdu, Y., "Blade-Row Interactions in a Low Pressure Turbine at Design and Strong Off-Design Operation", Journal of Turbomachinery, Vol. 136, 2014. DOI: 10.1115/1.4028213

[19] Walther, B. and Nadarajah, S., "Adjoint-Based Constrained Aerodynamic Shape Optimization for Multistage Turbomachines", Journal of Propulsion and Power, Vol. 31, No. 5, 2015, pp. 1298-1319. DOI: 10.2514/1.B35433

[20] ANSYS Inc., ANSYS FLUENT 14.0, User's guide.

[21] Mieloszyk, J., Galinski, C. and Piechna, J., "Contrarotating propeller for fixed wing MAV: part 1", Aircraft Engineering and Aerospace Technology, Vol. 85, No. 4, 2013, pp. 304-315. DOI: 10.1108/AEAT-Jan-2012-0008

[22] Selig, M. S., Lyon, C. A., Giguere, P., Ninham, C. P. and Guglielmo, J. J., Summary of Low-Speed Airfoil Data (Volume 2), SoarTech Publications, Virginia Beach, Virginia, 1996.

[23] Selig, M. S. and Guglielmo, J. J., "High-Lift Low Reynolds Number Airfoil Design", Journal of Aircraft, Vol. 34, No. 1, 1997, pp. 72-79. DOI: 10.2514/2.2137

[24] Cummings, R. M., Forsythe, J. R., Morton, S. A. and Squires, K. D., "Computational Challenges in High Angle of Attack Flow Prediction", Progress in Aerospace Sciences, Vol. 39, No. 5, 2003, pp. 369-384. DOI: 10.1016/S03760421(03)00041-1 